\title{
Pioglitazone and bladder cancer risk: a multipopulation pooled, cumulative exposure analysis
}

\author{
Daniel Levin • Samira Bell • Reijo Sund • Sirpa A. Hartikainen • Jaakko Tuomilehto • \\ Eero Pukkala • Ilmo Keskimäki • Ellena Badrick • Andrew G. Renehan • Iain E. Buchan • \\ Samantha L. Bowker • Jasjeet K. Minhas-Sandhu • Zafar Zafari • Carlo Marra • Jeffrey A. Johnson • \\ Bruno H. Stricker • Andrè G. Uitterlinden • Albert Hofman • Rikje Ruiter • Catherine E. de Keyser • \\ Thomas M. MacDonald • Sarah H. Wild • Paul M. McKeigue • Helen M. Colhoun • \\ on behalf of the Scottish Diabetes Research Network Epidemiology \\ Group and the Diabetes and Cancer Research Consortium
}

Received: 14 July 2014 / Accepted: 3 November 2014 /Published online: 7 December 2014

(C) Springer-Verlag Berlin Heidelberg 2014

\begin{abstract}
Aims/hypothesis The evidence on the association between pioglitazone use and bladder cancer is contradictory, with many studies subject to allocation bias. The aim of our study was to examine the effect of exposure to pioglitazone on bladder cancer risk internationally across several cohorts. The potential for allocation bias was minimised by focusing on the cumulative effect of pioglitazone as the primary endpoint using a time-dependent approach.
\end{abstract}

Electronic supplementary material The online version of this article (doi:10.1007/s00125-014-3456-9) contains peer-reviewed but unedited supplementary material, which is available to authorised users.

D. Levin · H. M. Colhoun

Diabetes Epidemiology Group, Population Health Sciences,

University of Dundee, Dundee, UK

S. Bell $(\bowtie)$

Renal Unit, Ninewells Hospital \& Medical School,

Dundee DD1 9SY, UK

e-mail: samira.bell@nhs.net

R. Sund

Centre for Research Methods, Department of Social Research,

University of Helsinki, Helsinki, Finland

J. Tuomilehto

Hjelt Institute, Department of Public Health, University of Helsinki,

Helsinki, Finland

S. A. Hartikainen

School of Pharmacy, University of Eastern Finland, Kuopio, Finland

J. Tuomilehto

Department of Chronic Disease Prevention, National Institute for

Health and Welfare, Helsinki, Finland
Methods Prescription, cancer and mortality data from people with type 2 diabetes were obtained from six populations across the world (British Columbia, Finland, Manchester, Rotterdam, Scotland and the UK Clinical Practice Research Datalink). A discrete time failure analysis using Poisson regression was applied separately to data from each centre to model the effect of cumulative drug exposure on bladder cancer incidence, with time-dependent adjustment for ever use of

\footnotetext{
J. Tuomilehto

Center for Vascular Prevention, Danube University Krems, Krems, Austria

J. Tuomilehto

Diabetes Research Group, King Abdulaziz University, Jeddah, Saudi Arabia

E. Pukkala

Institute for Statistical and Epidemiological Cancer Research, Finnish Cancer Registry, Helsinki, Finland

I. Keskimäki

Division of Health and Social Services, National Institute for Health and Welfare, Helsinki, Finland

E. Pukkala • I. Keskimäki

School of Health Sciences, University of Tampere,

Tampere, Finland

E. Badrick

Institute of Population Health, University of Manchester,

Manchester, UK
} 
pioglitazone. These were then pooled using fixed and random effects meta-regression.

Results Data were collated on 1.01 million persons over 5.9 million person-years. There were 3,248 cases of incident bladder cancer, with 117 exposed cases and a median follow-up duration of 4.0 to 7.4 years. Overall, there was no evidence for any association between cumulative exposure to pioglitazone and bladder cancer in men (rate ratio [RR] per 100 days of cumulative exposure, $1.01 ; 95 \%$ CI $0.97,1.06$ ) or women (RR $1.04 ; 95 \%$ CI $0.97,1.11$ ) after adjustment for age, calendar year, diabetes duration, smoking and any ever use of pioglitazone. No association was observed between rosiglitazone and bladder cancer in men (RR 1.01; 95\% CI $0.98,1.03$ ) or women (RR 1.00; $95 \%$ CI 0.94, 1.07).

Conclusions/interpretation The cumulative use of pioglitazone or rosiglitazone was not associated with the incidence of bladder cancer in this large, pooled multipopulation analysis.

Keywords Bladder cancer · Epidemiology $\cdot$ Meta-analysis · Pioglitazone $\cdot$ Rosiglitazone $\cdot$ Thiazeolidinedione

$\begin{array}{ll}\text { Abbreviations } \\ \text { CPRD } & \text { Clinical Practice Research Datalink } \\ \text { FDA } & \text { Food and Drug Administration } \\ \text { PPAR } & \text { Peroxisome proliferator-activated receptor } \\ \text { RR } & \text { Rate ratio } \\ \text { TZD } & \text { Thiazolidinedione }\end{array}$

\section{Introduction}

Bladder cancer is the ninth most common cancer in the world, with 430,000 new cases diagnosed in 2012. Europe and North

\author{
A. G. Renehan \\ Faculty Institute of Cancer Sciences, University of Manchester, \\ Manchester, UK \\ I. E. Buchan \\ Farr Institute@ HeRC, University of Manchester, Manchester, UK
}

S. L. Bowker · J. K. Minhas-Sandhu $\cdot$ J. A. Johnson

School of Public Health, University of Alberta, Edmonton, AB,

Canada

Z. Zafari

Center for Clinical Epidemiology and Evaluation, Vancouver Coastal

Health Institute, Vancouver, BC, Canada

\section{Marra}

Faculty of Pharmaceutical Sciences, University of British Colombia, Vancouver, BC, Canada

B. H. Stricker · A. G. Uitterlinden - A. Hofman • C. E. de Keyser Department of Epidemiology, Erasmus MC, Rotterdam,

The Netherlands
America have the highest incidence of bladder cancer, with a higher incidence in those with diabetes $[1,2]$. Peroxisome proliferator-activated receptor gamma (PPAR $\gamma$ ) is a nuclear transcription factor which, in addition to being expressed in normal urothelium, is overexpressed in bladder tumours [3,4]. Pioglitazone and rosiglitazone are thiazolidinediones (TZDs) which act as PPAR $\gamma$ agonists and are used in the treatment of type 2 diabetes. Their use, however, has been questioned owing to safety concerns. In preclinical studies, exposure of male rats to pioglitazone was associated with an increased risk of bladder cancer [5]. In 2005, the ProActive trial (a large European randomised controlled trial evaluating the effects of pioglitazones on cardiovascular outcomes) found a nonsignificant increase in the incidence of bladder cancer in the pioglitazone-treated group: $14(0.5 \%)$ cases compared with 6 $(0.2 \%)$ in controls [6]. Re-analysis excluding cases occurring within 1 year of study so as to improve biological plausibility identified six cases in the pioglitazone group and two in the placebo group [7].

Based on these studies, the US Food and Drug Administration (FDA) commissioned the manufacturer to carry out a 10 year observational cohort study to further examine this risk. An initial mid-term analysis showed that two or more years of cumulative exposure to pioglitazone were associated with an increased risk of bladder cancer [8]. Around the same time, a similar increase in risk was observed in a French cohort [9], which led to the withdrawal of pioglitazone from France and Germany. The FDA issued a recommendation in 2010 that pioglitazone should be avoided in patients with bladder cancer and prescribed with caution in patients with previous bladder cancer [10]. The European Medicine Agency extended this to patients with uninvestigated macroscopic haematuria [11].

B. H. Stricker · A. G. Uitterlinden

Department of Internal Medicine, Erasmus MC, Rotterdam,

The Netherlands

B. H. Stricker • C. E. de Keyser

Inspectorate of Health Care, The Hague, the Netherlands

R. Ruiter

Department of Internal Medicine, Groene Hart Ziekenhuis,

Gouda, the Netherlands

T. M. MacDonald

Medicines Monitoring Unit (MEMO), Division of Cardiovascular \& Diabetes Medicine, Ninewells Hospital \& Medical School, Dundee, UK

S. H. Wild • P. M. McKeigue

Centre for Population Health Sciences, University of Edinburgh,

Edinburgh, UK

H. M. Colhoun

Public Health, NHS Fife, Kirkcaldy, UK 
Since these cautions, several studies and meta-analyses of published data have reported contradictory results [12-24]. This may result from issues associated with observational study design, including limited sample size and a lack of attention to potential biases such as cancer ascertainment bias, immortal time bias, and the effects of diabetes duration and glycaemic control $[25,26]$. There are only two reported cohorts with over 1,000 cases of bladder cancer; therefore, achieving an adequate sample size can be a challenge in observational studies $[9,16]$. Allocation bias is very difficult to eradicate from observational pharmacoepidemiology studies, even with extensive covariate adjustment and propensity score analyses. Those who are given a prescription can differ from those who are not in subtle unmeasured ways which can be related to the risk of the outcome. We previously argued that analysis of the cumulative effect of a drug on outcome in models that also include time-dependent covariate adjustment for ever vs never use is one way to avoid such between-person allocation bias [27]. While some of the analyses on pioglitazone until now have reported bladder cancer risks by increasing cumulative exposure, they did so by reporting rate ratios (RR)s compared with those who were never exposed $[8,9$, 16]. This approach, however, does not prevent allocation bias.

The aim of our study was to address the limitations of previous observational studies by measuring the effect of exposure to pioglitazone on bladder cancer risk across several cohorts. This was done by pooling data to increase sample size in an aggregate-level analysis using the same methodology in which we explicitly set out to focus on cumulative effects as the primary exposure, adjusting for ever usage in a timedependent approach.

\section{Methods}

Overview Analyses were conducted in six populations (British Columbia, Finland, Manchester, Rotterdam, Scotland and the UK Clinical Practice Research Datalink [CPRD]), using identical methods for formatting and analysing the data; results were combined using fixed effects meta-regression. By specifying a common data format, preparing generic scripts to be run by each centre to both analyse its data and obtain sufficient statistical output for the metaanalysis, de facto data sharing was achieved while governance requirements were satisfied. Ethical approval was obtained by each centre from their respective authorities.

Selection of participants and person time for inclusion into the models In each centre, anyone with type 2 diabetes at any time since the introduction of TZDs in that centre (circa 1999) was considered for inclusion. Thus, the analysis included both prevalent and incident cases of diabetes. There was no age restriction for inclusion in the study. Anyone known to have had bladder cancer prior to the entry date of diagnosis of diabetes was excluded and anyone whose cumulative exposure to TZDs could not be evaluated was excluded. That is, persons were excluded if they had diabetes at any time from the date of TZD introduction onwards but had a period of diabetes of 1 year or more when their drug data were not captured. In practice, this means that all TZD users in the analysis were incident or very recent users. If diabetes ascertainment was unclear, then at least 1 year of diabetes from the presumed date of onset without being on any glucose-lowering drugs had to have elapsed to be considered evaluable for subsequent cumulative exposure to TZD or other drugs. This condition was set to limit these study entrants to probable cases of incident diabetes. For models in which we further adjusted for other glucose-lowering drugs, anyone whose cumulative exposure to those drugs could not be evaluated was excluded. In practice, this means that anyone with diabetes preceding the availability of their drug data by 1 year or more was excluded from these models, but those with retrospective data or drug exposure throughout their diabetes were included.

Using prescription data, we defined all periods of exposure to TZD, metformin, insulin and sulfonylurea for each patient. We defined follow-up for the analysis as the date of introduction of TZDs in that centre until the latest date for which data were available. For each individual the date of entry into the analysis was the latest of the following three dates: the first date under observation for drug prescribing, the date of diabetes diagnosis or the date of TZD introduction at that centre. The exit date was defined as the earliest of the date of event, the date of death, the last date under observation for drug prescribing or the last date under observation for cancer events.

Data preparation for modelling and calculation of exposure The follow-up period was divided into discrete intervals of 28 days, with occurrence of a cancer of interest in any interval being scored as a binary variable. A data matrix was generated, with one row for each individual under observation and columns specifying event status (coded as binary) at end and covariate values at the beginning of each 28 day interval. A series of models were constructed, each with a minimal-to-full set of covariates. Covariates consisted of sex, age, diabetes duration, calendar year, BMI, smoking (current, past or never), and both time updated ever exposure and cumulative exposure to each drug of interest. For each drug class, cumulative exposure at the start of each interval was calculated as the sum of all earlier intervals in which a drug prescription was current. The exposure duration was calculated from the first and last dates of a run of repeat prescriptions at a given dose plus the duration of the last prescription in that run. Gaps between runs of repeat prescriptions did not accumulate exposure. Following drug cessation, the cumulative number of days of exposure at cessation was carried forward in subsequent time intervals. 
Events Cancer data were obtained from either validated cancer registries based on primary care read codes or clinician validated review of pathology and medical records (see electronic supplementary material [ESM] Methods). Bladder neoplasms ranged from malignant neoplasm of the bladder, carcinoma in situ and neoplasms of uncertain behaviour. Our prespecified primary endpoint was malignant neoplasm using WHO ICD-10 (www.who.int/classifications/icd/en/) codes C67.0-C67.9. Only the first event was considered.

Statistical methods The effects of cumulative exposure were evaluated using a discrete time survival model $[28,29]$. This was set up as a Poisson regression model in which each individual contributed one observation for each 28 day interval between their entry and exit dates. The model included age, diabetes duration, calendar year, and cumulative and ever exposure times to one or more drugs under study. Some models were further adjusted for BMI and smoking (where these data were available). The main cumulative effect measure was assumed to be linear. An alternative categorical variable for pioglitazone cumulative exposure $(<1,1-2$ and $\geq 2$ years) was also examined. The models were run separately for each sex. Although there is no evidence to suggest that other glucoselowering drugs are causal or protective in bladder cancer, we considered it important to rule out any potential confounding by other glucose-lowering drugs since the prevalence of their use varies between those ever and never exposed to TZDs. We also therefore evaluated the effects of other glucose-lowering drugs and covariates by including terms for ever exposure and time-dependent cumulative exposure to sulfonylureas, metformin and insulin, as well as time-dependent terms for time since diagnosis of diabetes. One way to be certain that an estimate of a drug's causal effect cannot be due to such confounding is to model the data with two time updated terms in the model: a binary term for ever exposure up to that time point and a continuous term for cumulative exposure. The ever exposure term contrasts the rate in unexposed person time periods with the predicted rate in person time periods when exposure has just started. It is the sum of any stepwise (immediate) effect of drug exposure on the event, the confounding effect of timeinvariant frailty (i.e. allocation bias) and any reverse causality (where effects of an as-yet undiagnosed tumour leads to the drug of interest being prescribed for the first time). Inclusion of the ever exposure term results in the cumulative exposure term being independent of unexposed person time intervals within the likelihood equation, and is therefore unaffected by allocation bias. We then focused the inference of causality on the cumulative term [27].

Meta-analytic method Using R Metafor software (www. metafor-project.org/doku.php/installation), a fixed effects model was applied to the individual centre coefficients for ever and cumulative exposure effects. Heterogeneity of effects was measured using the $\mathrm{Q}$ test to determine the need for a random effects model, which was performed where the $p$ value was $\leq 0.05$.

Sensitivity analysis Sensitivity of the results was checked by running three groups of models with nested covariates. In addition, the main exposure of interest, pioglitazone, was compared with an equivalent model in which it was replaced with the other commonly used TZD, rosiglitazone. Further models used lagged exposure times (1 and 2 year lagging) and expanded neoplasm categories: all three neoplasm ICD-10 codes C67, D09.0 and D41.1 for the bladder and the combination of malignant neoplasms of bladder, ureter and renal pelvis. Additional analyses combined models that included adjustment for smoking in centres where smoking data were available (complete in Manchester, Rotterdam and Scotland; unavailable in British Columbia and Finland). To further investigate the potential confounding of pioglitazone exposure with smoking (due to smoking data being unavailable in British Colombia and Finland), we specified an analysis with lung cancer as the outcome (ICD-10 C34.0-C34.9). Since pioglitazone exposure is not thought to cause lung cancer, any association of ever or cumulative exposure to pioglitazone with lung cancer incidence would indicate that smoking might confound other pioglitazone outcome associations. Given the size of the CPRD dataset and that events in that dataset were not from cancer registry data, we conducted a sensitivity analysis excluding that dataset.

\section{Results}

Descriptive data Data were collected over all centres for 1.01 million persons over 5.9 million person-years. Characteristics for individuals at study entry for each centre are shown in Table 1 . The median age at entry in each centre varied from 60 to 64 years across the cohorts. All centres had the same difference in median age at entry between persons ever exposed and never exposed to pioglitazone; those ever exposed had a median age at entry 6 years lower than those never exposed (not shown). Table 2 summarises the person time and bladder cancer events and rates for each centre by sex and pioglitazone exposure status: $3.7 \%$ of person-years and 3.6\% of the events occurred after exposure to pioglitazone. The median follow-up duration varied from 4.0 to 7.4 years (Table 2). There were 3,248 incident bladder cancer (ICD-10 C67) events and 5,262 bladder neoplasms (ICD-10 C67, D09.0 and D41.4). The overall incidence of bladder cancer ranged from 46.4 to 80.8 cases per 100,000 person-years. Rates were substantially lower in women than in men (29.0/ 100,000 person-years at risk vs $90.7 / 100,000$ person-years at risk). Overall, there were just 117 bladder cancers and 204 bladder neoplasms in those ever exposed to pioglitazone by 
Table 1 Patient characteristics by country/region at study entry

\begin{tabular}{|c|c|c|c|c|c|c|}
\hline Characteristic & Scotland & CPRD & Finland & British Columbia & Rotterdam & Manchester \\
\hline Total number & 252,269 & 156,443 & 426,767 & 153,862 & 6,694 & 11,561 \\
\hline Age $(\text { years })^{\mathrm{a}}$ & $63.9(54.5-72.5)$ & $64.0(54.4-73.0)$ & $63.8(53.7-73.2)$ & $61.0(51.4-71.1)$ & $63.9(54.9-72.4)$ & $60.0(50.3-68.6)$ \\
\hline Females (\%) & $116,963(46)$ & $71,521(46)$ & $211,010(49)$ & $71,582(47)$ & $3,350(50)$ & $5,105(44)$ \\
\hline DM duration (years) ${ }^{\mathrm{a}}$ & $0(0-1.5)$ & $0(0-0)$ & $0(0-3.8)$ & $0(0-1.61)$ & $0.5(0-3.5)$ & $0(0-1.2)$ \\
\hline Entry (year) ${ }^{\mathrm{a}}$ & $2002(2001-2006)$ & 2006 (2003-2009) & 2003 (2001-2008) & $2001(2000-2004)$ & $2001(2001-2004)$ & 2003 (2001-2007) \\
\hline BMI $\left(\mathrm{kg} / \mathrm{m}^{2}\right)^{\mathrm{a}}$ & $30.1(26.7-34.4)$ & $30.2(26.7-34.6)$ & NA & NA & $30(26-33.9)$ & $30.2(26.8-34.6)$ \\
\hline Current smokers (\%) & $58,243(23)$ & $26,487(17)$ & NA & NA & $2,180(33)$ & $2,758(24)$ \\
\hline $\begin{array}{l}\text { Participants with }>1 \text { year } \\
\text { DM duration at entry }(\%)\end{array}$ & 28 & 10 & 34 & 30 & 42 & 26 \\
\hline
\end{tabular}

${ }^{\mathrm{a}}$ Median and interquartile range

DM, diabetes mellitus

the end of follow-up. There were 22 ever exposed bladder cancers in 22,505 patient-years, with a cumulative exposure of $2-3$ years and 10 cases in 26,004 patient-years with a cumulative exposure of $>3$ years.

The exact date of diabetes diagnosis was known for $61 \%$ of participants. Models adjusting for smoking and BMI among its covariates excluded these variables for cohorts from Finland and British Columbia because no data on BMI or smoking were available for these centres. Thus, only partial adjustment for smoking could be achieved.

Meta-analyses Figures 1 and 2 show forest plots by centre, with an overall estimate of the cumulative effect of pioglitazone on bladder cancer. Figure 1 is adjusted for age, calendar year and ever exposure to pioglitazone. Figure 2 includes further adjustment for diabetes duration and smoking (where available). The direction of effect varied between populations but was not significant within any population; overall, there was no evidence of any association. The additional covariate adjustment made no difference to these results (Fig. 2). Furthermore, there was no evidence for an association with ever exposure in men or women (Fig. 3). Further adjustment for prior exposure to other glucose-lowering drugs in the populations in which these data were available did not alter the RR for cumulative exposure or ever vs never exposure to pioglitazone: men, RR per 100 days cumulative exposure 1.00 $(95 \%$ CI $0.95,1.05)$ pre- and post-adjustment; women, RR per 100 days cumulative exposure $1.03(95 \%$ CI $0.96,1.11)$ preadjustment, 1.03 (95\% CI 0.95, 1.10) post-adjustment. There was no evidence for an association between cumulative exposure to any other class of diabetes drug and bladder cancer (data not shown). Prior exposure to other glucose-lowering drugs was lower in the Manchester cohort than in other centres. However, omitting this centre from the metaanalysis had no impact on the results.

A model in which linear pioglitazone exposure was replaced with categorical cumulative exposure $(<1,1-2$ and
$>2$ years) gave men RRs of 1.52 (95\% CI $0.92,2.52)$ for $1-$ 2 vs $<1$ year cumulative exposure, and 1.27 (95\% CI 0.74 , 2.16) for $>2$ vs $<1$ year. For women, the RRs were $2.1(95 \%$ CI $0.56,7.77)$ and $2.36(95 \%$ CI $0.72,7.72)$ for $1-2$ and $>2$ years, respectively.

The same trends were obtained in the sensitivity analysis excluding CPRD data. Sensitivity analyses combining all malignant neoplasms of the bladder, carcinoma in situ of the bladder and neoplasms of uncertain behaviour of the bladder did not show any significant effects of cumulative days of exposure (RR per 100 days in men 1.01 [95\% CI 0.98, 1.04]) or ever use vs never use of pioglitazone (RR per 100 days in men 1.19 [95\% CI 0.94, 1.51]). Further sensitivity analyses combining malignant neoplasm of bladder, ureter and renal pelvis did not reveal any associations (data not shown).

There was no evidence for any residual confounding by smoking in that there were no significant cumulative or ever exposure effects in the lung cancer model without smoking adjustment: RR $0.99(95 \%$ CI $0.96,1.03)$ for male cumulative exposure and RR 1.01 (95\% CI 0.98, 1.04) for female cumulative exposure.

Q tests showed evidence of heterogeneity in the effects of cumulative and ever exposure to pioglitazone in some of the models (see Figs 1 and 2 for cumulative exposure models in men). In cases with heterogeneity, random effects models showed that significance and conclusions were unchanged. Models were repeated to evaluate rosiglitazone exposure (Fig. 4). There was no significant effect of rosiglitazone exposure on bladder cancer.

\section{Discussion}

In this pooled, aggregate-level analysis across multiple populations in several different countries, we found no significant association between cumulative exposure to pioglitazone and 
Table 2 Person time and bladder cancer events and rates for each dataset by sex and pioglitazone exposure status

\begin{tabular}{|c|c|c|c|c|c|c|c|}
\hline Variable & Scotland & CPRD & Finland & British Columbia & Rotterdam & Manchester & All \\
\hline $\begin{array}{l}\text { Follow-up } \\
\left(_{\text {years })^{\mathrm{a}}}\right.\end{array}$ & $5.7(2.8-9.4)$ & $5.2(2.5-8.1)$ & $5.1(2.3-9.5)$ & $4.0(1.8-6.0)$ & $5.4(2.8-8.2)$ & $7.4(3.6-10.5)$ & $\mathrm{NA}^{\mathrm{b}}$ \\
\hline \multicolumn{8}{|l|}{ Men } \\
\hline$n(\%)$ & $135,306(25.6)$ & $84,922(16.1)$ & 215,757 (40.9) & $82,280(15.6)$ & $2,917(0.6)$ & $6,456(1.2)$ & $527,638(100.0)$ \\
\hline \multicolumn{8}{|c|}{ Events $(\%)$} \\
\hline Total & $566(22.6)$ & $478(19.1)$ & $1,046(41.8)$ & $382(15.3)$ & $4(0.2)$ & $25(1.0)$ & $2,501(100.0)$ \\
\hline $\mathrm{NE}$ & $543(22.5)$ & $447(18.5)$ & $1,028(42.6)$ & $367(15.2)$ & $4(0.2)$ & $24(1.0)$ & $2,413(100.0)$ \\
\hline $\mathrm{EE}$ & $23(26.1)$ & $31(35.2)$ & $18(20.5)$ & $15(17.0)$ & $0(0.0)$ & $1(1.1)$ & $88(100.0)$ \\
\hline \multicolumn{8}{|c|}{ Patient-years (\%) } \\
\hline Total & $750,503(27.2)$ & $463,146(16.8)$ & $1,172,961(42.5)$ & $311,881(11.3)$ & $15,930(0.6)$ & $42,619(1.5)$ & $2,757,040(100.0)$ \\
\hline $\mathrm{NE}$ & $711,917(26.9)$ & $430,869(16.3)$ & $1,142,875(43.2)$ & $305,647(11.5)$ & $15,303(0.6)$ & $40,547(1.5)$ & $2,647,158(100.0)$ \\
\hline $\mathrm{EE}$ & $38,586(35.1)$ & $32,276(29.4)$ & $30,086(27.4)$ & $6,234(5.7)$ & $627(0.6)$ & $2,072(1.9)$ & $109,882(100.0)$ \\
\hline \multicolumn{8}{|c|}{ Events per 100,000 person-years $(95 \% \mathrm{CI})$} \\
\hline Total & $75.4(69.3,84.1)$ & $103.2(94.2,117)$ & $89.2(83.9,96)$ & $122.5(110.5,136.8)$ & $25.1(6.8,65.6)$ & $58.7(38,88.8)$ & $90.7(87.2,96.3)$ \\
\hline $\mathrm{NE}$ & $76.3(70,83)$ & $103.7(94.3,113.8)$ & $89.9(84.5,95.6)$ & $120.1(108.1,133)$ & $26.1(7.1,66.9)$ & $59.2(37.9,88.1)$ & $91.2(87.6,94.9)$ \\
\hline Rate EE & $59.6(37.8,89.4)$ & $96(65.3,136.3)$ & $59.8(35.5,94.6)$ & $240.6(134.7,396.9)$ & $0(0,588)$ & $48.3(1.2,268.9)$ & $80.1(64.2,98.7)$ \\
\hline \multicolumn{8}{|l|}{ Women } \\
\hline$n(\%)$ & $116,963(24.4)$ & $71,521(14.9)$ & $211,010(44)$ & $71,582(14.9)$ & $2,926(0.6)$ & $5,105(1.1)$ & $479,107(100)$ \\
\hline \multicolumn{8}{|c|}{ Events $(\%)$} \\
\hline Total & $231(30.9)$ & $121(16.2)$ & $283(37.9)$ & $90(12.0)$ & $11(1.5)$ & $11(1.5)$ & $747(100.0)$ \\
\hline $\mathrm{NE}$ & $216(30.1)$ & $112(15.6)$ & $280(39.0)$ & $89(12.4)$ & $10(1.4)$ & $11(1.5)$ & $718(100.0)$ \\
\hline $\mathrm{EE}$ & $15(51.7)$ & $9(31.0)$ & $3(10.3)$ & $1(3.4)$ & $1(3.4)$ & $0(0.0)$ & $29(100.0)$ \\
\hline \multicolumn{8}{|c|}{ Patient-years (\%) } \\
\hline Total & $659,105(25.6)$ & $395,198(15.3)$ & $1,199,613(46.6)$ & $272,136(10.6)$ & $15,822(0.6)$ & $34,899(1.4)$ & $2,576,773(100)$ \\
\hline $\mathrm{NE}$ & $626,114(25.1)$ & $369,985(14.9)$ & $1,177,338(47.3)$ & $267,959(10.8)$ & $15,238(0.6)$ & $33,503(1.3)$ & $2,490,135(100)$ \\
\hline $\mathrm{EE}$ & $32,991(38.1)$ & $25,214(29.1)$ & $22,275(25.7)$ & $4,177(4.8)$ & $585(0.7)$ & $1,396(1.6)$ & $86,638(100)$ \\
\hline \multicolumn{8}{|c|}{ Events per 100,000 person-years $(95 \% \mathrm{CI})$} \\
\hline Total & $35(30.7,40.9)$ & $30.6(25.4,37.8)$ & $23.6(20.9,26.8)$ & $33.1(26.6,41)$ & $69.5(34.7,126.7)$ & $31.5(15.7,57.5)$ & $29(26.9,31.7)$ \\
\hline $\mathrm{NE}$ & $34.5(30.1,39.4)$ & $30.3(24.9,36.4)$ & $23.8(21.1,26.7)$ & $33.2(26.7,40.9)$ & $65.6(31.5,120.7)$ & $32.8(16.4,58.7)$ & $28.8(26.8,31)$ \\
\hline $\mathrm{EE}$ & $45.5(25.4,75)$ & $35.7(16.3,67.8)$ & $13.5(2.8,39.4)$ & $23.9(0.6,133.4)$ & $171(4.3,952.9)$ & $0(0,264.2)$ & $33.5(22.4,48.1)$ \\
\hline \multicolumn{8}{|l|}{ Both sexes } \\
\hline$n(\%)$ & $252,269(25.1)$ & $156,443(15.5)$ & $426,767(42.4)$ & $153,862(15.3)$ & $5,843(0.6)$ & $11,561(1.1)$ & $1,006,745(100.0)$ \\
\hline \multicolumn{8}{|c|}{ Events (\%) } \\
\hline Total & $797(24.5)$ & $599(18.4)$ & $1,329(40.9)$ & $472(14.5)$ & $15(0.5)$ & $36(1.1)$ & $3,248(100.0)$ \\
\hline $\mathrm{NE}$ & $759(24.2)$ & $559(17.9)$ & $1,308(41.8)$ & $456(14.6)$ & $14(0.4)$ & $35(1.1)$ & $3,131(100.0)$ \\
\hline $\mathrm{EE}$ & $38(32.5)$ & $40(34.2)$ & $21(17.9)$ & $16(13.7)$ & $1(0.9)$ & $1(0.9)$ & $117(100.0)$ \\
\hline \multicolumn{8}{|c|}{ Patient-years (\%) } \\
\hline Total & $1,409,608(26.4)$ & $858,344(16.1)$ & $2,372,574(44.5)$ & $584,017(10.9)$ & $31,752(0.6)$ & $77,517(1.5)$ & $5,333,813(100.0)$ \\
\hline $\mathrm{NE}$ & $1,338,031(26)$ & $800,854(15.6)$ & $2,3202,13(45.2)$ & $573,606(11.2)$ & $30,540(0.6)$ & $74,049(1.4)$ & $5,137,293(100.0)$ \\
\hline $\mathrm{EE}$ & $71,578(36.4)$ & $57,490(29.3)$ & $52,361(26.6)$ & $10,411(5.3)$ & $1,212(0.6)$ & $3,468(1.8)$ & $196,520(100.0)$ \\
\hline \multicolumn{8}{|c|}{ Events per 100,000 person-years $(95 \% \mathrm{CI})$} \\
\hline Total & $56.5(52.7,60.6)$ & $69.8(64.3,75.6)$ & $56(53,59.1)$ & $80.8(73.7,88.5)$ & $47.2(26.4,77.9)$ & $46.4(32.5,64.3)$ & $60.9(58.8,63)$ \\
\hline $\mathrm{NE}$ & $56.7(52.8,60.9)$ & $69.8(64.1,75.8)$ & $56.4(53.4,59.5)$ & $79.5(72.4,87.1)$ & $45.8(25.1,76.9)$ & $47.3(32.9,65.7)$ & $60.9(58.8,63.1)$ \\
\hline $\mathrm{EE}$ & $53.1(37.6,72.9)$ & $69.6(49.7,94.7)$ & $40.1(24.8,61.3)$ & $153.7(87.8,249.6)$ & $82.5(2.1,459.7)$ & $28.8(0.7,160.7)$ & $59.5(49.2,71.4)$ \\
\hline
\end{tabular}

${ }^{a}$ Median and interquartile range

${ }^{\mathrm{b}} \mathrm{NA}$ : median follow-up for all centres combined is not available, as datasets kept separate $\mathrm{EE}$, ever exposed to pioglitazone; NE, never exposed to pioglitazone 
Fig. 1 Association of cumulative days of exposure (per 100 days) to pioglitazone with bladder cancer incidence within centres and combined across centres in (a) men and (b) women, with adjustment for age, calendar year, and ever exposure to pioglitazone ( $\log _{e}$-transformed axis and whiskers). (a) The absence of ever exposure events in Rotterdam prevented convergence and hence is excluded from the plot. (b) The absence of ever exposure events in Manchester prevented convergence and hence is excluded from the plot. B. Columbia, British Columbia; Exp., exposed; FE, fixed effects; RE, random effects; Unexp., unexposed

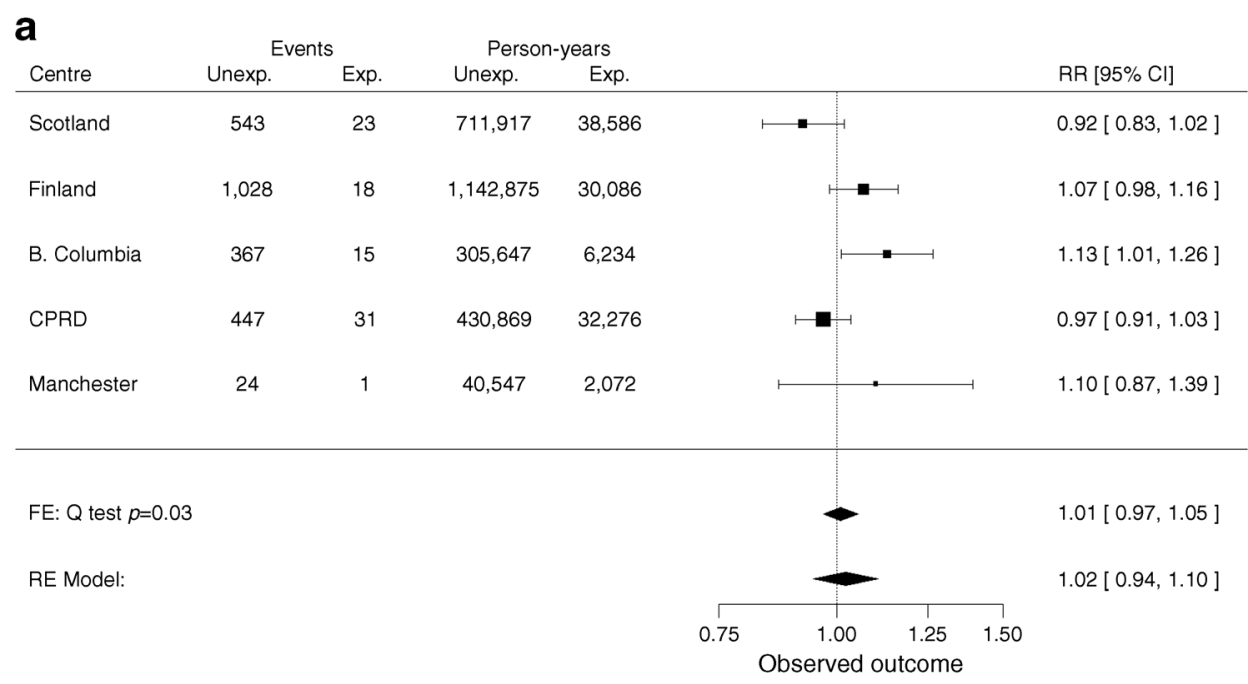

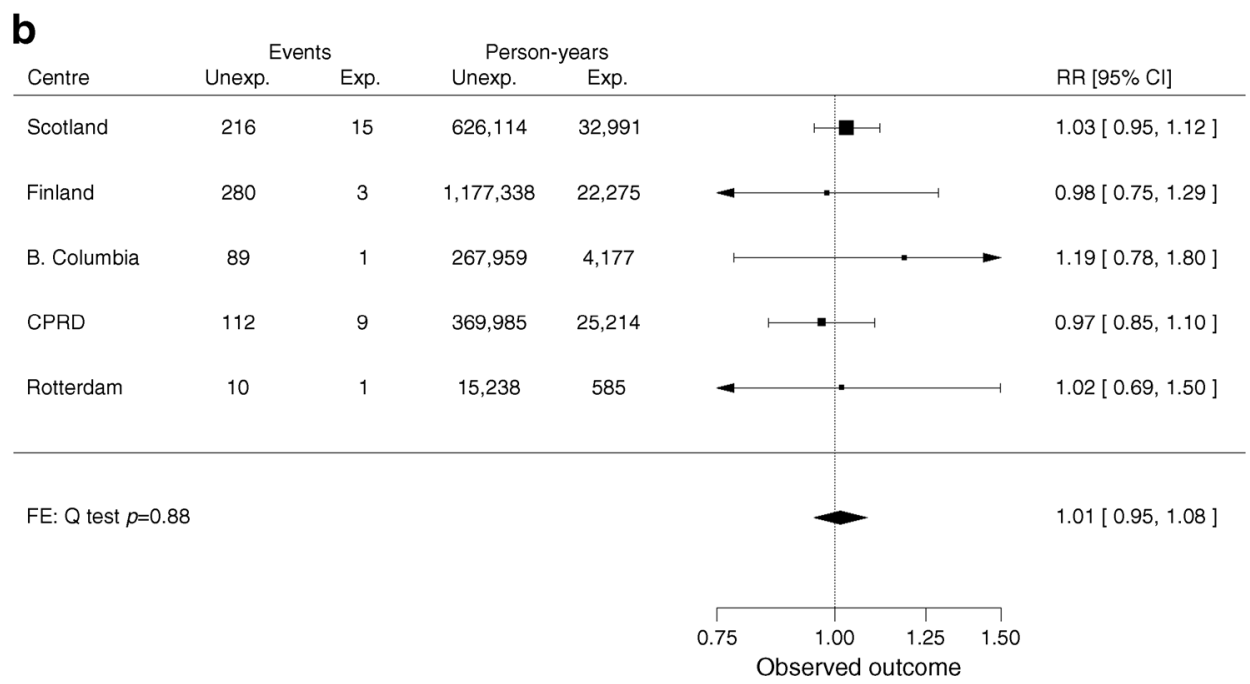

bladder cancer when using methods to minimise allocation bias. The contradictory directions of ever exposure effects between centres suggest inter-centre differences in the profiles of patients treated with the drug. In addition, there was no relationship between rosiglitazone and bladder cancer.

Our study has a number of strengths. Most observational pharmacoepidemiological studies are limited by allocation bias. Comparisons of event rates between ever users and never users of a drug are likely to be confounded by 'frailty', that is, those prescribed and those not prescribed the drug may differ in their prior susceptibility to bladder cancer. Such 'allocation bias' or 'confounding by indication' cannot be reliably dealt with by simple adjustment for measured covariates such as past medical history. Another important aspect of our analysis is that we considered exposure to other glucose-lowering drugs in the models, something that many other studies have not done.
Alternatively, examining cumulative effects of pioglitazone in exposed persons only would avoid allocation bias. However, this would preclude the simultaneous evaluation of several exposures to glucose-lowering drugs with different start dates, as well as discarding information relevant to other covariate effects. Our approach of focusing on cumulative exposure but using time-dependent adjustment for ever usage avoids between-person allocation bias while maximising the use of covariate data. It also allowed us to check whether exposure to any other glucoselowering drug might confound an association between pioglitazone and bladder cancer. A further important advantage of our approach, which contrasts with previous studies, is that we included data from a number of countries worldwide using the same methodology to analyse the data from each countries, thereby increasing the size of our cohort, while limiting heterogeneity. 
Fig. 2 Association of cumulative days of exposure (per 100 days) to pioglitazone with bladder cancer incidence within centres and combined across centres in (a) men and (b) women, with adjustment for age, calendar year, diabetes duration, smoking (where available) and ever exposure to pioglitazone $\left(\log _{e^{-}}\right.$ transformed axis and whiskers).

(a) The absence of ever exposure events in Rotterdam prevented convergence and hence is excluded from the plot. (b) The absence of ever exposure events in Manchester prevented convergence and hence is excluded from the plot. B. Columbia, British Columbia; Exp., exposed; FE, fixed effects; RE, random effects; Unexp., unexposed

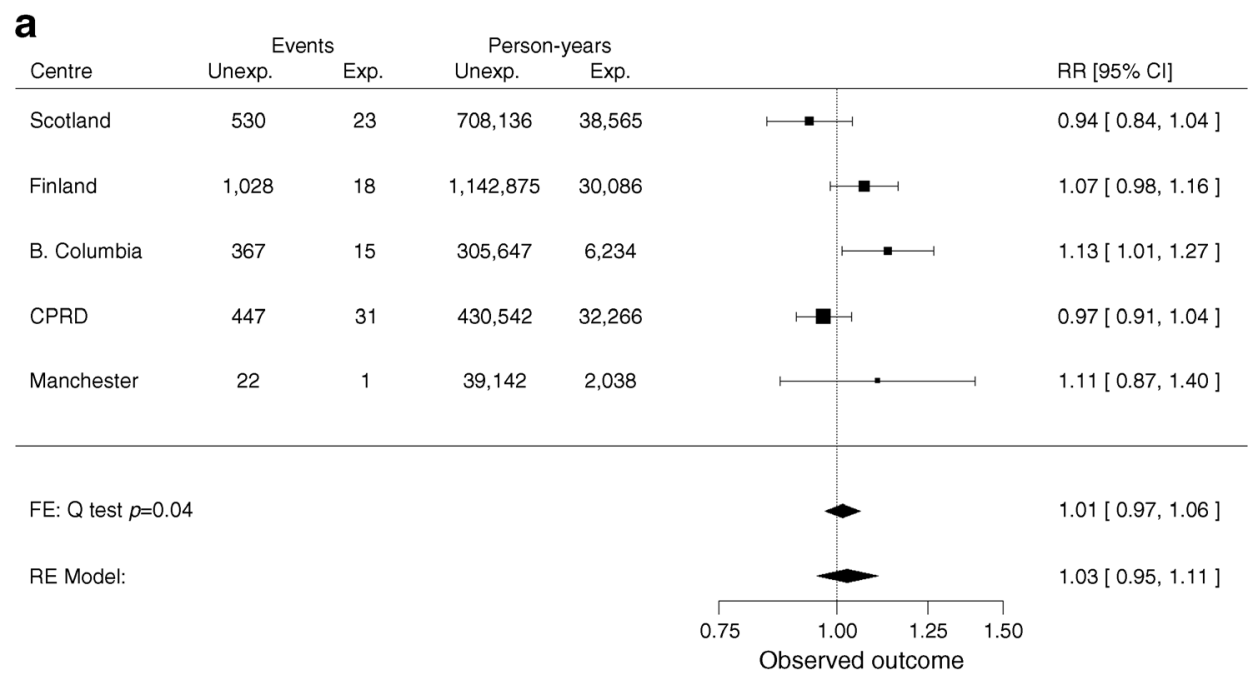

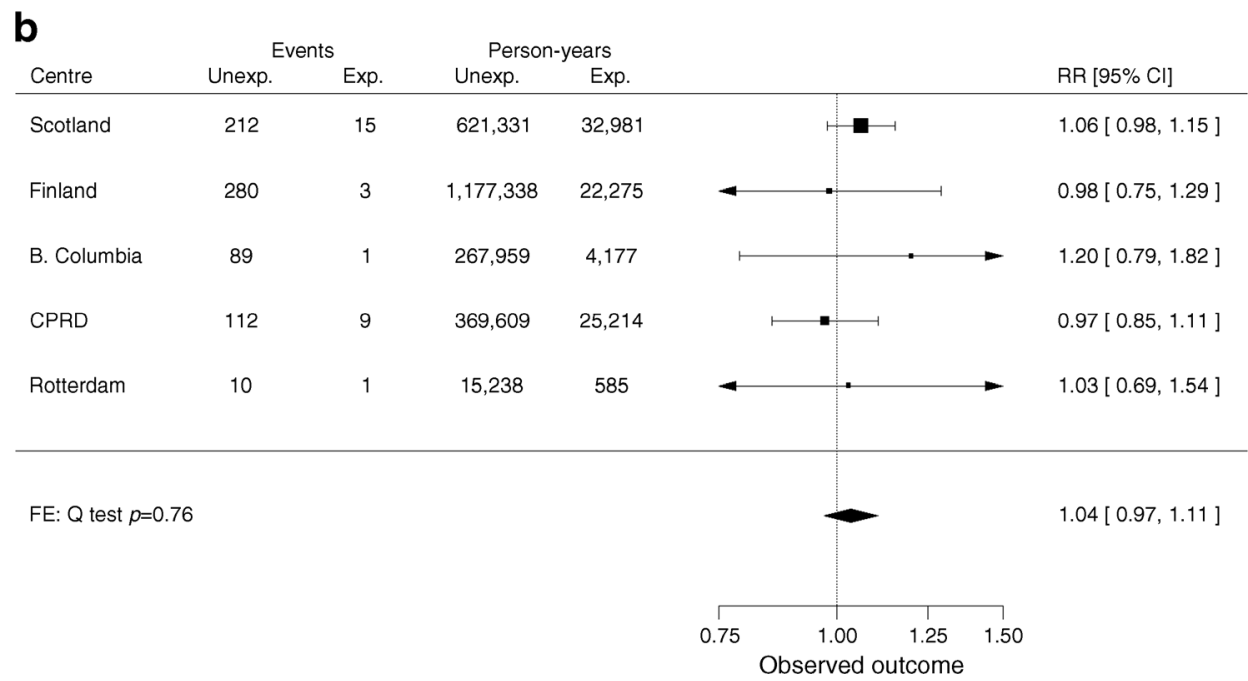

Limitations of our study include the short follow-up time. Our study includes more exposed cases compared with other reports, but the actual numbers remain relatively small. Compared with other reported observational studies, our cohort had a long follow-up (4.0-7.4 years), but of course further studies involving a longer exposure to pioglitazone would be desirable. However, to obtain robust results and allow for a reasonable interval between pioglitazone exposure and cancer development, we consider that a 10 year follow-up would be needed to rule out an association. A further limitation is that our data are mainly limited to white Europeans, with an absence of other ethnicities, and there is a lack of complete data on BMI and smoking across the centres. In addition, we did not include a French population in our study as our aim was to replicate previous findings made in a French population in other cohorts. We were not able to adjust for proteinuria testing, which may lead to detection bias [30], nor for confounders such as kidney disease and urinary tract infection [31]. A further minor limitation was the possibility that the same individuals were included in both the CPRD and
Manchester cohorts (Scottish data was removed from CPRD data). However, removing the Manchester cohort from the meta-analysis had no impact on the results.

The rates of bladder cancer observed in our study were in keeping with the international statistics on population rates of bladder cancer [32]. The higher incidence in British Columbia compared with the European centres is consistent with the known higher rates in North America compared with Europe [2]. Observational studies examining the risk of bladder cancer and pioglitazone have yielded contradictory results. These studies varied in size and design, but all were prone to inappropriate causal inference and potential confounding. Table 3 summarises the characteristics of such studies. Most include a small number of exposed cases. Three studies include a comparable number of exposed cases to ours, but have contradictory results $[8,9,16]$. Neumann et al reported that pioglitazone was significantly associated with a risk of bladder cancer in men in a French cohort study. This cohort included almost 1.5 million diabetic patients from France, with 175 cases of bladder cancer in patients exposed to pioglitazone and a 
Fig. 3 Association of ever exposure to pioglitazone with bladder cancer incidence within centres and combined across centres in (a) men and (b) women, with adjustment for age, calendar year, diabetes duration, smoking (where available) and cumulative exposure to pioglitazone $\left(\log _{e}\right.$-transformed axis and whiskers). (a) The absence of ever exposure events in Rotterdam prevented convergence and hence is excluded from the plot. (b) The absence of ever exposure events in Manchester prevented convergence and hence is excluded from the plot.

B. Columbia, British Columbia; FE, fixed effects a

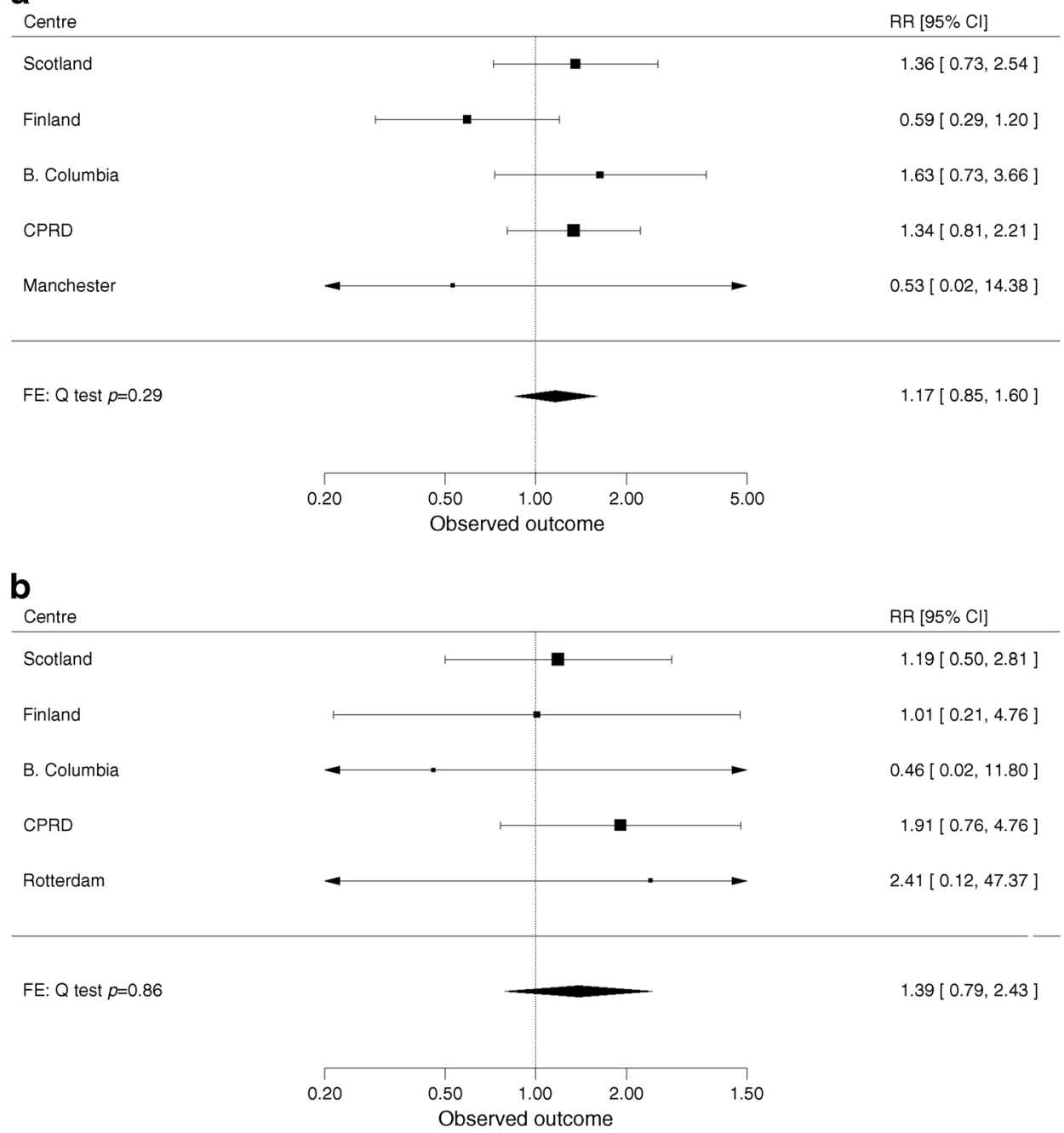

Table 3 Published observational studies (excluding meta-analyses) examining an association between pioglitazone and bladder cancer

\begin{tabular}{lllll}
\hline Reference & Country & Year & $\begin{array}{l}\text { Exposed } \\
\text { cases }(n)\end{array}$ & $\begin{array}{l}\text { Total } \\
\text { cases }(n)\end{array}$ \\
\hline Current study & $\begin{array}{l}\text { Canada, Finland, } \\
\text { the Netherlands, UK }\end{array}$ & 2014 & 117 & 3,248 \\
Kuo et al [17] & Taiwan & 2014 & 15 & 259 \\
Dormandy et al [6] & European & 2005 & 14 & 20 \\
Tseng [21] & Taiwan & 2012 & 10 & 165 \\
Lewis et al [8] & USA & 2011 & 90 & 881 \\
Hsiao et al [16] & Taiwan & 2013 & 153 & 3,412 \\
Neumann et al [9] & France & 2012 & 175 & 2,016 \\
Romero et al [35] & USA & 2014 & 9 & 204 \\
Wei et al [23] & UK & 2012 & 66 & 869 \\
Piccinni et al [19] & USA ${ }^{\text {a }}$ & 2011 & 31 & 93 \\
Azoulay et al [12] & UK & 2012 & 19 & 470 \\
Fujimoto et al [15] & Japan & 2013 & 9 & 682 \\
Song et al [20] & Korea & 2012 & 21 & 329 \\
\hline
\end{tabular}

${ }^{\mathrm{a}}$ Commissioned by the FDA median follow-up of 3.1 years [9]. In our study, both male and female effects varied between centres, with no clear trend. Furthermore, the study by Neumann et al used never exposed participants as the reference group and did not adjust for smoking. In a Taiwanese nested case-control study, both pioglitazone and rosiglitazone were significantly associated with bladder cancer. This study included 3,412 cases of bladder cancer, with 153 patients exposed to pioglitazone. The mean follow-up was 3.6 years. Both pioglitazone and rosiglitazone were associated with an increased risk of bladder cancer; the risk increased with longer exposure to these drugs. However, most studies reported no association between rosiglitazone and bladder cancer, and a recent meta-analysis showed no pooled effect [33]. This finding, together with the use of never exposed participants as the reference group (and its inclusion in the test for trend), suggests that these results may be due to allocation bias. Once again, no adjustment was made for smoking. However, a mid-term analysis of an FDAcommissioned study of the Kaiser Permanente Northern California diabetes registry at 5 years showed no 
Fig. 4 Association of cumulative days of exposure (per 100 days) to rosiglitazone with bladder cancer incidence within centres and combined across centres in (a) men and (b) women, with adjustment for age, calendar year and ever exposure to rosiglitazone $\left(\log _{e}\right.$-transformed axis and whiskers). (b) The absence of ever exposure events in Manchester prevented convergence and hence is excluded from the plot. B. Columbia, British Columbia; Exp., exposed; FE, fixed effects; Unexp., unexposed

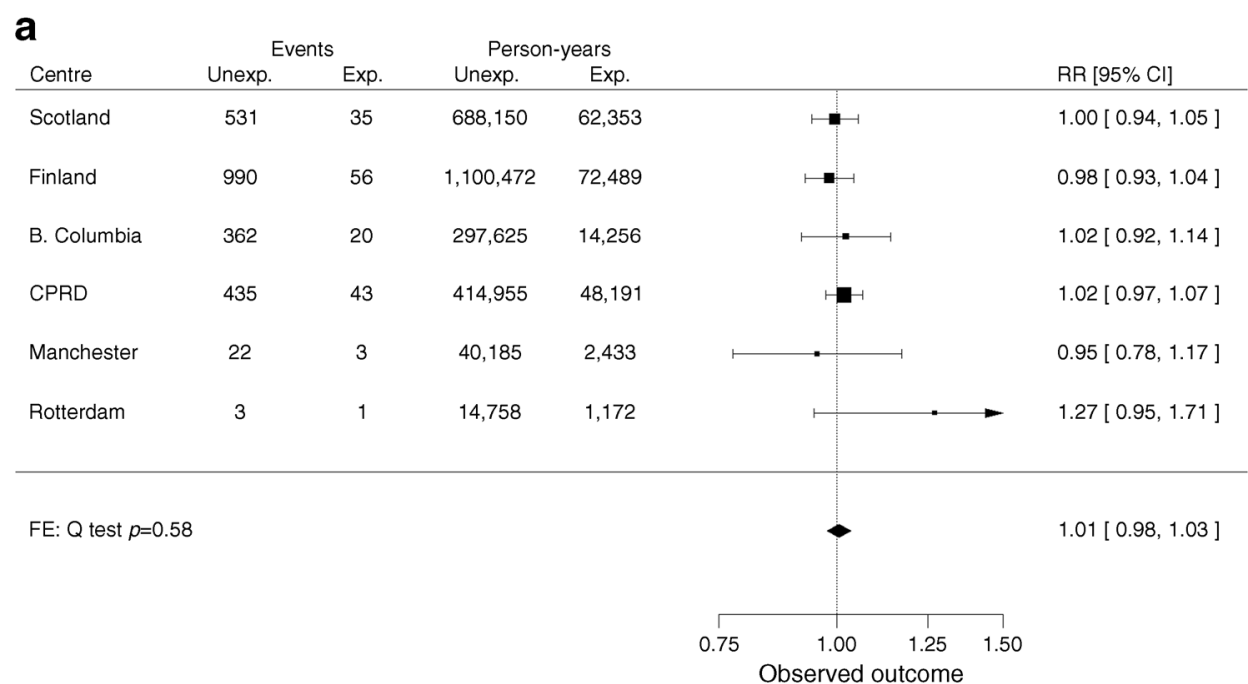

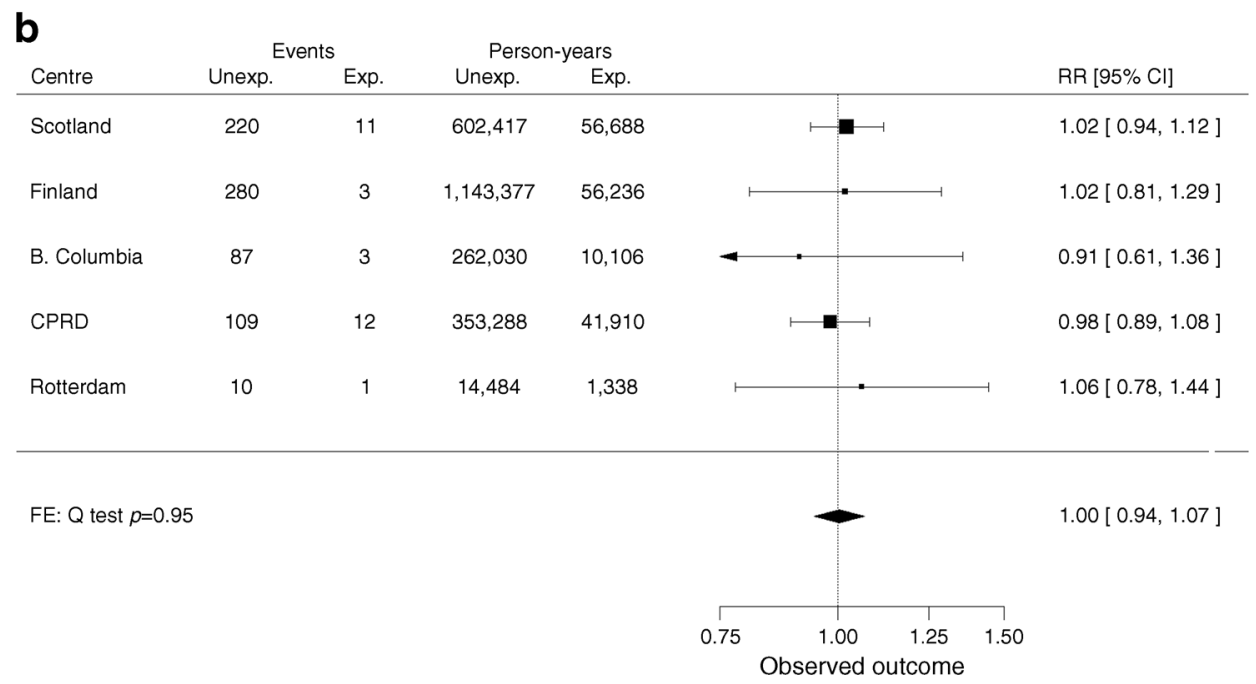

association between bladder cancer and short-term pioglitazone use, but the risk increased slightly with the longest exposure period and highest cumulative dose [8]. This study included 193,099 cases, with 90 exposed patients. The authors recently suggested that proteinuria testing may have had a confounding effect in this cohort. They found that patients treated with pioglitazone were more likely than others to undergo proteinuria testing. They hypothesised that this could lead to early detection of asymptomatic haematuria, and thereby earlier bladder cancer detection [30]. Although these large studies measured cumulative exposure, they are still prone to allocation bias because they used never exposed patients as the control group.

In addition to these single centre studies, there have been several meta-analyses of published data, which reported an increased risk of bladder cancer with pioglitazone use [13, 24, 33]. These are limited by the heterogeneity of the study methodology and the different combinations of confounders measured.
This analysis, to our knowledge, is the only one to use identical methodology across international centres involving a large number of diabetic patients. We have shown that even when the same methods are used, the ever use vs never use relationship varies between centres. This emphasises the high potential for allocation bias when using analyses that simply compare users with ever users and never users, and offers a possible explanation for the contradictory results in previous studies.

In summary, our large international analysis does not support a causal effect of pioglitazone on bladder cancer, thus contradicting previous studies deemed to have proven this relationship [30, 34]. To fully resolve this controversy, future analyses are needed, involving longer follow-up of exposed persons and using methods to minimise allocation bias.

Acknowledgements We wish to thank T. Akbar, Diabetes Epidemiology Unit, University of Dundee, UK, for her assistance in preparing this manuscript. 
Funding This study was funded by the European Foundation for the Study of Diabetes.

Duality of interest SHW, CM, JAJ, SLB, RS, RR, SAH, ZZ, DL, SB, BHS, AGU, AH, CEdK, EP, IK, IB, EB and JKM-S have no conflict of interests to declare. JT has received research support from AstraZeneca, Boehringer Ingelheim, Merck Sharp \& Dohme, Novartis, Pfizer, Sanofi and Servier, and has acted as a consultant, advisory board member or speaker for Bayer Health Care, Impeto Medical, Novartis, Novo Nordisk, Sanofi, Eli Lilly, Merck Serono and Merck Sharp \& Dohme. TMM has no direct competing interests in regards to this manuscript. His department holds research grants from Novartis, Pfizer, Amgen, Ipsen, Teijin and Menarini. He is and has been the principal investigator on trials paid for by Pfizer, Novartis, Ipsen, Teijin and Menarini. In the last 5 years, he has been paid consulting or speaker fees by Pfizer, Novartis, Kaiser Permanante, Takeda, Servier, Shire, Astellas, Menarini, and AstraZeneca. AGR has received honoraria and independent research grants from Novo Nordisk. HMC has received research support from Roche, Pfizer, Eli Lilly, Boehringer Ingelheim and AstraZeneca. She has also been part of the speaker's bureau and acted as a consultant for Pfizer. She is on an advisory panel for Sanofi, Pfizer, Novartis Pharmaceuticals and Eli Lilly, and owns Roche stocks and shares. None of these activities directly relate to this manuscript. PMM is a stakeholder of Pharmatics.

Contribution statement All authors made substantial contributions to the conception and design of the various aspects of the prospective studies or to the acquisition, analysis or interpretation of data. All authors also contributed to drafting the article or revising it critically for important intellectual content and have given final approval of the version to be published. HMC is responsible for the integrity of this work as a whole.

\section{References}

1. Larsson SC, Orsini N, Brismar K, Wolk A (2006) Diabetes mellitus and risk of bladder cancer: a meta-analysis. Diabetologia 49:28192823

2. Ferlay J SI, Ervik M, Dikshit R, Eser S, Mathers C, Rebelo M, Parkin DM, Forman D, Bray, F. Cancer incidence and mortality worldwide: IARC CancerBase No. 11. Available from http://globocan.iarc.fr, accessed 13 Dec 2013

3. Yoshimura R, Matsuyama M, Segawa Y et al (2003) Expression of peroxisome proliferator-activated receptors (PPARs) in human urinary bladder carcinoma and growth inhibition by its agonists. Int $\mathrm{J}$ Cancer 104:597-602

4. Nakashiro KI, Hayashi Y, Kita A et al (2001) Role of peroxisome proliferator-activated receptor gamma and its ligands in nonneoplastic and neoplastic human urothelial cells. Am J Pathol 159: 591-597

5. Suzuki S, Arnold LL, Pennington KL et al (2010) Effects of pioglitazone, a peroxisome proliferator-activated receptor gamma agonist, on the urine and urothelium of the rat. Toxicol Sci 113:349-357

6. Dormandy JA, Charbonnel B, Eckland DJ et al (2005) Secondary prevention of macrovascular events in patients with type 2 diabetes in the PROactive Study (PROspective pioglitAzone Clinical Trial In macroVascular Events): a randomised controlled trial. Lancet 366: 1279-1289

7. Erdmann E, Dormandy JA, Massi-Benedetti M, Spanheimer R (2011) Pioglitazone and bladder cancer? Authors' reply. Lancet 378:1544-1545

8. Lewis JD, Ferrara A, Peng T et al (2011) Risk of bladder cancer among diabetic patients treated with pioglitazone: interim report of a longitudinal cohort study. Diabetes Care 34:916-922
9. Neumann A, Weill A, Ricordeau P, Fagot JP, Alla F, Allemand H (2012) Pioglitazone and risk of bladder cancer among diabetic patients in France: a population-based cohort study. Diabetologia 55: 1953-1962

10. Food and Drug Administration (2010) FDA drug safety communication: ongoing safety review of Actos (pioglitazone) and potential increased risk of bladder cancer after two years exposure. Available from www.fda.gov/drugs/drugsafety/ucm226214.htm, accessed 15 Jun 2014

11. European Medicines Agency (2011) European Medicines Agency recommends new contra-indications and warnings for pioglitazone to reduce small increased risk of bladder cancer. Available from www. ema.europa.eu/ema/index.jsp?curl=pages/news and events/news/ 2011/07/news_detail_001311.jsp\&mid=WC0b01ac058004d5c1, accessed 15 Jun 2014

12. Azoulay L, Yin H, Filion KB et al (2012) The use of pioglitazone and the risk of bladder cancer in people with type 2 diabetes: nested casecontrol study. BMJ 344:e3645

13. Colmers IN, Bowker SL, Majumdar SR, Johnson JA (2012) Use of thiazolidinediones and the risk of bladder cancer among people with type 2 diabetes: a meta-analysis. CMAJ 184:E675-E683

14. Ferwana M, Firwana B, Hasan R et al (2013) Pioglitazone and risk of bladder cancer: a meta-analysis of controlled studies. Diabet Med 30: 1026-1032

15. Fujimoto K, Hamamoto Y, Honjo S et al (2013) Possible link of pioglitazone with bladder cancer in Japanese patients with type 2 diabetes. Diabetes Res Clin Pract 99:e21-e23

16. Hsiao FY, Hsieh PH, Huang WF, Tsai YW, Gau CS (2013) Risk of bladder cancer in diabetic patients treated with rosiglitazone or pioglitazone: a nested case-control study. Drug Saf 36:643-649

17. Kuo HW, Tiao MM, Ho SC, Yang CY (2014) Pioglitazone use and the risk of bladder cancer. Kaohsiung J Med Sci 30:94-97

18. MacKenzie T, Zens MS, Ferrara A, Schned A, Karagas MR (2011) Diabetes and risk of bladder cancer: evidence from a case-control study in New England. Cancer 117:1552-1556

19. Piccinni C, Motola D, Marchesini G, Poluzzi E (2011) Assessing the association of pioglitazone use and bladder cancer through drug adverse event reporting. Diabetes Care 34:1369-1371

20. Song SO, Kim KJ, Lee BW, Kang ES, Cha BS, Lee HC (2012) The risk of bladder cancer in Korean diabetic subjects treated with pioglitazone. Diabetes Metab J 36:371-378

21. Tseng CH (2012) Pioglitazone and bladder cancer: a populationbased study of Taiwanese. Diabetes Care 35:278-280

22. Tseng CH (2013) Rosiglitazone is not associated with an increased risk of bladder cancer. Cancer Epidemiol 37:385-389

23. Wei L, MacDonald TM, Mackenzie IS (2013) Pioglitazone and bladder cancer: a propensity score matched cohort study. Br J Clin Pharmacol 75:254-259

24. Zhu Z, Shen Z, Lu Y, Zhong S, Xu C (2012) Increased risk of bladder cancer with pioglitazone therapy in patients with diabetes: a metaanalysis. Diabetes Res Clin Pract 98:159-163

25. Tseng $\mathrm{CH}$ (2014) A review on thiazolidinediones and bladder cancer in human studies. J Environ Sci Health Part C Environ Carcinog Ecotoxicol Rev 32:1-45

26. Suissa S, Azoulay L (2012) Metformin and the risk of cancer: time-related biases in observational studies. Diabetes Care 35: 2665-2673

27. Colhoun HM, Livingstone SJ, Looker HC et al (2012) Hospitalised hip fracture risk with rosiglitazone and pioglitazone use compared with other glucose-lowering drugs. Diabetologia 55:2929-2937

28. Rodríguez G (2007) Lecture notes on generalized linear models. Available from http://data.princeton.edu/wws509/notes/, accessed 14 Jun 2014

29. Thompson WA Jr (1977) On the treatment of grouped observations in life studies. Biometrics 33:463-470 
30. Lewis JD, Habel L, Quesenberry C et al (2014) Proteinuria testing among patients with diabetes mellitus is associated with bladder cancer diagnosis: potential for unmeasured confounding in studies of pioglitazone and bladder cancer. Pharmacoepidemiol Drug Saf 2: 636-645

31. Tseng CH (2011) Diabetes and risk of bladder cancer: a study using the National Health Insurance database in Taiwan. Diabetologia 54: 2009-2015

32. Cancer Research UK. Bladder cancer risks and causes. Available from www.cancerresearchuk.org/cancer-help/type/ bladder-cancer/about/bladder-cancer-risks-and-causes, accessed 15 Jun 2014
33. Turner RM, Kwok CS, Chen-Turner C, Maduakor CA, Singh S, Loke YK (2014) Thiazolidinediones and associated risk of bladder cancer: a systematic review and meta-analysis. Br J Clin Pharmacol $78: 258-273$

34. Feeley J, Matsuyama K (2014) Takeda, Lilly jury awards $\$ 9$ billion over Actos risks. Available from www.bloomberg.com/news/2014 04-07/takeda-actos-jury-awards-6-billion-in-punitive-damages.html, accessed 15 Jun 2014

35. Romero V, Peyton C, Gray I, Hemal A, Terlecki R (2014) Pathology of bladder cancer among diabetic patients undergoing radical cystectomy with a history of pioglitazone (Actos) use. BMC Urol $14: 10$ 